\title{
Neuropsychiatric Aspects of Head Injury - An Overview
}

\author{
Dr. Soumitra Ghosh ${ }^{1}$, Dr. Sandipan Nayek ${ }^{2}$ \\ ${ }^{1}$ Associate Professor, Assam Medical College, Dibrugarh \\ ${ }^{2}$ Post Graduate Trainee, Assam Medical College, Dibrugarh
}

\section{INTRODUCTION}

Traumatic brain injury (TBI) refers to any external mechanical force acting on the brain which may cause temporary or permanent dysfunction. The prevalence of TBI is increasing day by day and giving rise to many post TBI neuropsychiatric sequelae.

\section{EPIDEMIOLOGY AND ETIOLOGY}

About 200 per 100000 population per year suffer a head injury ${ }^{1}$. In the majority of these the head injury is classified as mild ${ }^{2}$, yet perhaps as many as 100 per 100000 per year go on to suffer significant disability at 1 year ${ }^{3}$. Increased rates of TBI in developing parts of the world are due to high two wheeler usage and poor road safety conditions. The peak incidence is between the ages of 15 and 24 and older than the age of 64 years. Males are affected twice as often as females. Motor vehicle accidents (MVAs) account for the most frequent civilian cause of TBI followed by fall.

\section{COMPARATIVE NOSOLOGY}

IN ICD-10

F06 : Other mental disorders due to brain damage and dysfunction and to physical disease

F07 : Personality and behavioural disorder due to brain disease, damage and dysfunction

\section{IN DSM 5}

Major Neurocognitive Disorder due to Traumatic brain injury

294.10 : without behavioral disturbance
294.11 : with behavioral disturbance

331.83 : Mild Neurocognitive Disorder due to Traumatic brain injury

\section{TYPES OF HEAD INJURY}

(A) Depending on the integrity of meningeal covering -

> Closed head injury - Motor vehicle accident, Falls, Assault, Sport related.

> Penetrating injury - Missile wound, Blast injury.

(B) Depending on the Pathological consequence of head injury :

> Primary brain damage

> Secondary brain damage

(C) Neuropathological classification of head injury -

$>$ Focal lesion

$>$ Diffuse lesions

\section{PATHOPHYSIOLOGY OF HEAD INJURY}

> Loss of Consciousness and Coma - The trauma results in massive and rapid release of potassium into the extracellular space ${ }^{4}$. This is associated with excessive glutamate release.

> Diffuse Axonal Injury - Resulting in axonal swelling, lobulation, and ultimately transection with formation of the classic retraction ball. 
> Cerebral Oedema - Diffuse cerebral swelling is more common in children ${ }^{5}$. The raised intracranial pressure results in herniation of brain tissue through the tentorium and under the falx cerebri.

> Cerebral Anoxia - Derives from cerebral oedema, hypotension, blood loss, disturbances in regulation of the cerebral circulation, and ventilatory insufficiency in the acute stages.

> Neuronal Death - Necrotic cell death occurs due to the toxic effects of excitatory neurotransmitters, particularly glutamate ${ }^{6}$, mitochondrial dysfunction ${ }^{7}$, calcium activation of proteases and phospholipases, formation of toxic free radicals and low intracellular magnesium and immunological processes ${ }^{8}$, including activation of interleukins ${ }^{9}$. Apoptosis occurs due to alterations of the balance within the cell of pro- and anti-apoptotic pathways. Pro-apoptotic proteins are Bax and caspase, and the anti-apoptotic protein is Bcl.

> Effects on Cerebral Metabolism - Normal or low flow rates in the first 24 hours, succeeded by relative hyperaemia ('luxury' perfusion) that may persist for many days. Increased cerebral glucose uptake is found in the first 3-4 days after severe brain injury. Global reductions in blood flow after head injury are associated with worse outcome ${ }^{11}$.

> A $\boldsymbol{\beta}$ Peptide Deposition - Accumulation of $A \beta$ peptides, which are produced by cleavage of $\beta A P P$, aggregate to form $\boldsymbol{\beta}$-amyloid protein.

$>$ Neurobiological changes after TBI

\section{NEUROTRANSMITTER AND ENDOCRINE CHANGES}

1. Abnormality in Glutamate Pathway-Glutamate concentration is increased in CSF several days after TBI.

2. Abnormality in Cholinergic Pathway Cholinergic neuronal activity is increased immediately after TBI, but later the activity is decreased.
3. Abnormality in Biogenic Amine Pathway - There is increased serotonergic and noradrenergic metabolites level in CSF.

Clinical Features - Clinical Features of Neuropsychiatric aspects of TBI can be divided into-

1) Acute effects of head injury

2) Chronic Sequelae

Acute Effects of Head Injury - 1) Impairment / Loss of Consciousness 2) Post Traumatic Delirium / Confusional state 3) Post traumatic agitation 4) Post Traumatic Amnesia (PTA).

Chronic Sequelae of Head Injury - Depends on multiple factors like mental constitution and premorbid personality, age at the time of injury, circumstances of the injury, compensation, litigation, secondary gain and attribution bias, development of epilepsy, amount of brain damage incurred, alcohol and drug abuse, location of brain damage incurred.

\section{POST-HEAD INJURY NEUROPSYCHIATRIC MANIFESTATIONS INCLUDE}

1) Cognitive Impairment - Post TBI cognitive impairment is pronounced in cases with PTA> $24 \mathrm{hrs}$. Dysexecutive syndrome may occur which may be tested by 'frontal lobe function' like The Behavioural Assessment of Dysexecutive Syndrome (BADS), Wisconsin Card Sorting Test (WCST), Trails B test, Verbal fluency test, Stroop test. .

2) Personality Changes - Frontal lobe syndrome is the classic example characterized by irritability, apathy, euphoria, disinhibition, inappropriate jocularity and altered sexual behavior. The case report of Phineas Gage as described by his doctor, John Harlow, is a prototype of post TBI personality change ${ }^{13}$. Based on the exact location in frontal lobe, various syndromes are described including DLPFC Syndrome aka Dysexecutive syndrome, OFC Syndrome 
aka Psuedopsychopathic, VMPFC Syndrome aka Psudeodepressive syndrome. Kretschmer described the injury to the basal parts of the brain as 'basal syndrome' which results from lesions of the midbrain, hypothalamus and orbitofrontal cortex.

3) Aggression - The predictors of aggression in TBI includes - alcohol, younger age at injury, being depressed, frontal injury, a pre-injury history of antisocial behaviour. The term organic aggression syndrome and 'episodic dyscontrol syndrome' has also been used. Characteristic features of organic aggression syndrome ${ }^{14}$ : Reactive : triggered by modest or trivial stimuli, Non-reflective : usually does not involve premeditation or planning, Non-purposeful : aggression serves no obvious long-term aims or goals, Explosive, Periodic : long periods of relative calm, Ego-dystonic : afterwards patients show remorse and are upset; behaviour is out of character; do not blame others or justify behaviour .

4) Psychosis - Characterized by paranoid delusion and auditory hallucinations. Factors distinguishing TBI psychosis from schizophrenia are the absence of Schneiderian first rank symptoms such as thought insertion and thought withdrawal, a later age of onset, less premorbid psychiatric disturbance, briefer duration, less common family history, better response to neuroleptics, less need for maintenance medication, and a better prognosis.

5) Affective/MoodDisorders-IncludesDepression and Mania. Major depression seen shortly after head injury was due to lesion location, whereas later-onset depression was more closely tied to psychosocial factors ${ }^{15}$. Kreutzer et al. ${ }^{16}$ found that the most frequent symptoms of depression were fatigue $(46 \%)$, frustration $(41 \%)$ and poor concentration (38\%).

6) Anxiety Disorders - It includes Generalised Anxiety Disorder (GAD), Phobic disorders, Posttraumatic stress disorder (PTSD), Obsessive- compulsive disorder (OCD), conversion disorder.

7) Post Concussion Syndrome - A clinical syndrome characterised by immediate and transient impairment of neural function, such as alteration of consciousness, disturbances of vision, equilibrium due to mechanical forces.

8) Post TBI Headache - Severe post TBI headache should raise suspicion of chronic $\mathrm{SDH}$ and requires full and careful neurological examination, supplemented by CT or MRI.

9) Punch-drunk Syndrome/Dementia Pugilistica - Multiple traumatic brain injury associated with boxing characterised by documented progressive dementia due to diffuse injury to the cortex, basal ganglia. Pathologically, dementia pugilistica shares many characteristics with Alzheimer's disease (i.e., neurofibrillary tangles, diffuse amyloid plaques, and/or tau immunoreactivity).

10) TBI as a risk factor for Epilepsy - Post TBI epilepsy develops in around 5\% of closed injuries and around $30 \%$ in penetrating injuries. Cortical scarring due to contusions are highly epileptogenic.

11) TBI as a risk factor for Dementia - Multiple mild TBIs are a definite risk factor for Dementia.

12) Suicide - Death by suicide occurs in about $1 \%$ of patients over the first 15 years after injury ${ }^{17}$. In the case of suicides, lesions were commonly found in the frontal and temporal lobes of the brain. Risk factors for suicide attempts include a post-injury history of psychiatric/emotional disturbance or substance abuse and clinically significant levels of suicide ideation ${ }^{18}$.

\section{INVESTIGATIONS FOR TBI}

1. Neuroimaging - Computed Tomography (CT), magnetic resonance imaging (MRI), Functional MRI (fMRI), Positron Emission Tomography (PET) (which shows level of cerebral metabolic activity), Single photon emission computerized 
tomography (SPECT) which shows level of cerebral perfusion, magnetic resonance spectroscopy (MRS) which shows areas of cerebral neuronal loss and diffusion tensor imaging and fiber tractography (DTI/FT) which shows cerebral white matter tracts.

2. Electrophysiological : Electroencephalography (EEG) is used to assess for post traumatic epilepsy or encephalopathy. Quantitative EEG (QEEG) is sometimes used for slow wave abnormality following TBI \& post traumatic temporal lobe epilepsy. Evoked response potentials (ERPs) and Video EEG monitoring or 24hrs ambulatory EEG is also useful. Polysomnography is helpful to assess atypical sleep disturbances following TBI like atypical night terror, sleep apnoea, nocturnal myoclonus.

3. Neuropsychological Testing : done to assess cognitive functioning, language testing, tests of motivation and malingering, tests for premorbid functioning.

\section{Blood Biochemistry}

> Serum Electrolytes $-\mathrm{Na}+, \mathrm{K}+, \mathrm{Mg}_{2}+, \mathrm{Cl}-$

> Neuronal Protein - include both neuronal (e.g., neuron-specific enolase, creatine kinase-BB, cleaved Tau protein) and glial (e.g., myelin basic protein, S-100B) proteins. Most sensitive serum marker is S-100B.

> Neuro Endocrine Assessment - for pituitary hormone deficiencies, Diabetes insipidus, syndrome of growth hormone deficiency (GHD), partial central hypothyroidism, low cortisol levels and decreased gonadotropin secretion.

\section{CLINICAL INDICATORS OF HEAD INJURY SEVERITY}

Major Indicators - Depth of unconsciousness as assessed by GCS , Duration of loss of consciousness / coma, Evidence of behavioural/cognitive change in the immediate period suggestive of delirium,
Neurological symptoms and signs, Evidence of skull fracture and/or other abnormalities on neuroimaging, Blood in the CSF, Duration of PTA.

Less useful indicators - Duration of retrograde amnesia, Abnormalities on EEG, Markers of cell damage (e.g. S-100B), Evidence of injury to head, e.g. lacerations, bruising, bleeding from ears, fracture of maxilla/zygoma.

\section{SEVERITY RATINGS FOR TBI}

Prognosis and Outcome - The long-term outcome of TBI patients is primarily related to severity of brain injury, type and location of intracranial lesion, patients' age, efficacy of acute medical and surgical treatment, socioeconomic status, educational level, previous psychiatric disorders (e.g., history of alcohol and/or drug abuse, personality disorders), premorbid social functioning levels, the quality and extent of rehabilitation services and the availability of social and vocational support.

Glasgow Outcome Scale (GOS) - The Glasgow Outcome Scale (GOS) has been widely used as a measure of the long-term outcome of TBI patients. It consists of five levels of outcome: (1) death, (2) persistent vegetative state, (3) severe disability (conscious but dependent in activities of daily living, (4) moderate disability (disabled but living independently), and (5) good recovery (mild neuropsychiatric effects but able to resume an otherwise normal life).

\section{Management 1) Pharmacotherapy}

\section{2) Rehabilitation and psychological therapies}

Pharmacotherapy - Drugs should be chosen with less potential for lowering seizure threshold, less extrapyramidal and anticholinergic side effects and less potential for drug interactions .

Cognitive Impairment - Psychostimulants and other dopaminergically active agents (e.g., methylphenidate, dextroamphetamine, 
amantadine, pergolide, and bromocriptine) may modestly improve arousal and speed of information processing, reduce distractibility, and improve some aspects of executive function. Cholinesterase inhibitors such as donepezil, mostly at $10 \mathrm{mg} /$ day has also positive effect on cognitive functioning, motivation, and general well being. Rivastigmine has also been found to be effective ${ }^{19}$.

Aggression, Agitation and Impulsion Antipsychotic and Anticonvulsants like Valproate, gabapentin, carbamazepine are useful. Sexually disinhibited behaviour may respond to antipsychotic medication. Sometimes an anti-androgen is needed $^{20}$.

Mania and Psychosis - Of the mood stabilisers, the anticonvulsant valproate is likely to be the first option when treating mania. Some patients will require an antipsychotic, in which case it is probably best to choose an atypical antipsychotic which should be started with low doses. Risperidone is effective, but olanzapine, quetiapine and aripiprazole are reasonable alternatives. Clozapine is used in patients who have failed to respond to other antispychotics and whose behaviour remains very difficult to manage ${ }^{21}$.

Depression and Anxiety Disorders - The selective serotonin reuptake inhibitors (SSRIs) are the first choice. Moclobemide and Trazodone are other alternatives. Electroconvulsive therapy is not contraindicated when other measures have failed ${ }^{22}$.

Post-traumatic headache - Valproate, Propranolol and/or Amitriptyline and Sumatriptan are useful. Non-addictive analgesics and Ergotamine preparations may be tried in vascular headache ${ }^{23}$ Antidepressants can sometimes produce results.

Management of Insomnia - For nocturnal insomnia, good sleep hygiene measures need to be taken. Treatment with hypnotics like benzodiazepine should be given cautiously. The non-benzodiazepine hypnotics like zopiclone, zolpidem are probably a better starting point. Sedative antidepressants may be considered, particularly trazodone. Melatonin and low dose amitriptyline $(25 \mathrm{mg})$ can be used in patients with chronic insomnia after head injury ${ }^{24}$ For severe daytime sleepiness modafinil seems safe and may be appropriate.

Head injury in Children - ADHD symptoms in children with head injury improved with methylphenidate treatment. Amantadine may improve behaviour and executive function ${ }^{25}$

\section{REHABILITATION AND PSYCHOLOGICAL THERAPIES :}

Early Rehabilitation for Severe Injury - Physical activity should be encouraged and the value of early mobilisation has come to be generally recognised. Graduated exercises and games help to restore the patient's physical self-confidence.

Rehabilitation for Neurological Sequelae - The main areas that require evaluation are locomotion, upper extremity function and impairment of communication. Hemiparesis, paraparesis or ataxia of gait requires physiotherapy. Occupational therapy has a special place in restoring useful function to the upper limbs. Speech therapy has role in helping the resolution of dysphasia or dysarthria.

Rehabilitation for Cognitive Impairment - The programme must be graded, with goals at any stage that are realisable, rational and acceptable to the patient ${ }^{26}$. Self-esteem is to be boostered.

\section{Components of Rehabilitation Programmes -} These include assessment, formulation of plan, implementation of plan, review and modification of plan and discharge arrangement.

Core rehabilitation Team - It should include neurologists, psychiatrists, rehabilitation physicians, clinical psychologists, physiotherapists, occupational therapists, speech therapists and psychiatric social workers.

Conclusion - Traumatic brain injury can cause not only focal deficits of motor activity or language, but also a variety of potentially disabling psychiatric symptoms and syndromes. These include mood and anxiety disorders; personality disturbances; 


\section{Neuropsychiatric Aspects of Head Injury - An Overview}

aggression; and psychosis. Treatment is complicated by cognitive deficits, lack of motivation, and lack of awareness of deficits. Pharmacological treatment may include a wide range of medications, such as antidepressants, antipsychotics, mood stabilizers, and stimulants. Family and individual counselling is particularly important in helping the patient and the family reconcile themselves to the reality of the behavioural changes in the patient post TBI.

\section{REFERENCES}

1. Bruns T.J., Hauser, W.A., The epidemiology of traumatic brain injury : a review. Epilepsia. 2003; 44 (suppl. 10) : 2-10.

2. Sorenson S.B., Kraus J.F. Occurrence, severity, and outcomes of brain injury. Journal of Head Trauma Rehabilitation; 1991; $6: 1-10$.

3. Thornhill S., Teasdale G.M. et al. Disability in young people and adults one year after head injury : prospective cohort study. British Medical Journal. 2003; 320 : 1631-1635.

4. Maroon J.C., Lovell M.R. et al.Cerebral concussion in athletes : evaluation and neuropsychological testing. Neurosurgery. 2000; 47 : 659-669.

5. Kazan S., Tuncer R. et al. Post-traumatic bilateral diffuse cerebral swelling. Acta Neurochirurgica (Wien). 1997; 139 : 295-301.

6. Faden A.I., Demediuk P. et al. The role of excitatory aminoacids and NMDA receptors in traumatic brain injury. Science. 1989; 244 : 798-800.

7. Robertson C.L. Mitochondrial dysfunction contributes to cell death following traumatic brain injury in adult and immature animals. Journal of Bioenergetics and Biomembranes. 2004; 36 : 363-368.

8. Schmidt O.I., Heyde C.E. et al. Closed head injury: an inflammatory disease? Brain Research Reviews. 2005; 48 : 388-399.

9. Tasci A., Okay O. et al.Prognostic value of interleukin-1 beta levels after acute brain injury. Neurological Research. 2003; $25: 871-874$.

10. Hausmann R., Biermann T. et al. Neuronal apoptosis following human brain injury. International Journal of Legal Medicine. 2004; 118 : 32-36.

11. Bouma G.J., Muizelaar J.P. et al. Cerebral circulation and metabolism after severe traumatic brain injury: the elusive role of ischemia. Journal of Neurosurgery. 1991; 75 : 685-693.

12. Alderso A.L., Novack T.A. Measuring recovery of orientation during acute rehabilitation for traumatic brain injury : value and expectations of recovery. Journal of Head Trauma Rehabilitation. 2002; 17 : 210-219.

13. Harlow, J.M. Recovery from the passage of an iron bar through the head. Publications of the Massachussetts Medical Society. 1868; 2 : 327-347. Cited in Damasio, H., Grabowski, T., Frank R. et al. The Return of Phineas Gage : clues about the brain from the skull of a famous patient. Science. 1994; 264 : 1102-1105.

14. Silver J.M., Yudofsky S.C. et al. (2005) Aggressive disorders. In: Silver, J.M., McAllister, T.W. \& Yudofsky, S.C. (eds) Textbook of Traumatic Brain Injury, pp. 259-278. American Psychiatric Publishing Inc., Washington, DC.

15. Jorge R.E., Robinson R.G. et al. Depression following traumatic brain injury : a 1 year longitudinal study. Journal of Affective Disorders. 1993; 27 : 233-243.

16. Kreutzer J.S., Seel R.T. et al. The prevalence and symptom rates of depression after traumatic brain injury : a comprehensive examination. Brain Injury. 2001; 15 : 563-576.

17. Tate R.L., Simpson G.K. et al. Completed suicide after traumatic brain injury. Journal of Head Trauma Rehabilitation. 1997; 12 : 16-28.

18. Simpson G. \& Tate R. Suicidality after traumatic brain injury: demographic, injury and clinical correlates. Psychological Medicine.2002; 32: 687-697.

19. Silver J.M., Koumaras B. et al. Effects of rivastigmine on cognitive function in patients with traumatic brain injury. Neurology. 2006; 67 : 748-755.

20. Emory L.E., Cole C.M. et al. Use of Depo-Provera to control sexual aggression in persons with traumatic brain injury. Journal of Head Trauma Rehabilitation. 1995; 10 : 47-58.

21. Michals M.L., Crismon M.L. et al. Clozapine response and adverse effects in nine brain-injured patients. Journal of Clinical Psychopharmacology. 1993; 13 : 198-203.

22. Kant R., Coffey C.E. et al. Safety and effi cacy of ECT in patients with head injury : a case series. Journal of Neuropsychiatry and Clinical Neuroscience. 1999; 11 : 32-37.

23. McBeath J.G. \& Nanda A. Use of dihydroergotamine in patients with postconcussion syndrome. Headache .1994;34: 148-151.

24. Kemp S., Biswas R. et al. The value of melatonin for sleep disorders occurring post-head injury : a pilot RCT. Brain Injury. 2004; 18 : 911-919.

25. Beers S.R., Skold A. et al. Neurobehavioral effects of amantadine after pediatric traumatic brain injury : a preliminary report. Journal of Head Trauma Rehabilitation. $2005 ; 20: 450-463$.

26. Wilson B.A., Evans J.J. et al. Cognitive rehabilitation : a goalplanning approach. Journal of Head Trauma Rehabilitation. 2002; 17 : 542-555. 